The Early Career Section offers information and suggestions for graduate students, job seekers, early career academics of all types, and those who mentor them. Angela Gibney serves as the editor of this section. Next month's theme will be advice from our advisor and publishing.

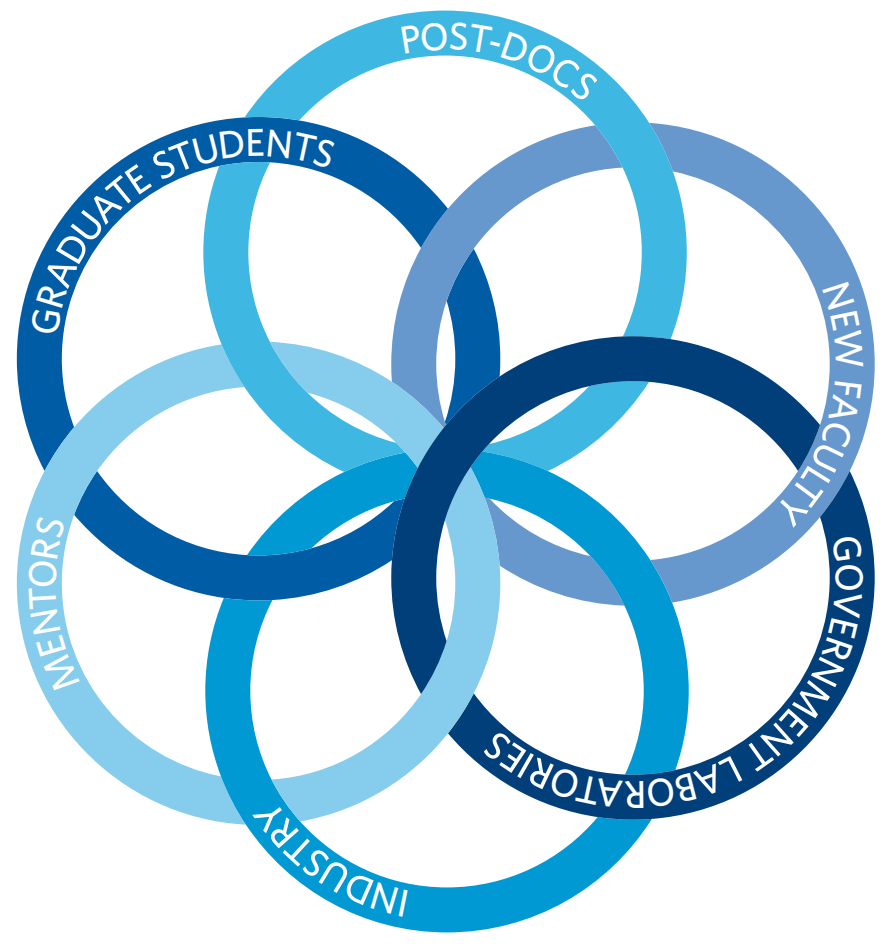

Online Resources

For permission to reprint this article, please contact: reprint-permission aams.org.

\section{How to Tutorial-a-thon}

\author{
Henry Adams, Hana Dal Poz \\ Kouřimská, Teresa Heiss, Sarah \\ Percival, and Lori Ziegelmeier
}

The opportunity. Each and every person in your research community has a mathematical topic that they explain well. Imagine if many such people each contributed a short tutorial video, describing a topic of their choosing, to a common YouTube channel. Could this make your research area more attractive to newcomers?

The challenge. Your challenge is to solicit, compile, moderate, and share high-quality videos that are contributed from members of your research community, in a manner that is time-efficient (for both you as the organizer and for the participants)! You want to encourage many people to contribute, including early-career researchers, instead of inviting only a handful of trusted content creators. Can you organize such an event in a way that doesn't require hundreds of emails or take over your semester?

Our response. Our response to this opportunity and challenge is the tutorial-a-thon. We organized our first tutorial-a-thon, for the applied topology community, during the month of March 2021. We ran a sequence of Zoom meetings (one per week), in which we (Week 1) explained the event and divided attendees into groups of size three; (Week 2) had attendees meet in their small groups in which each individual recorded their own trial-run tutorial of length $\leq 10$ minutes and received real-time feedback from their peers; and (Week 3) had attendees record their final tutorial, one video per person! This organization had the

Henry Adams is a professor of mathematics at Colorado State University. His email address is henry.adams@colostate.edu.

Hana Dal Poz Kourimská is a postdoctoral researcher in the Edelsbrunner research group at the Institute of Science and Technology Austria. Her email address is hana.kourimska@ist.ac.at.

Teresa Heiss is a PhD student in the Edelsbrunner research group at the Institute of Science and Technology Austria. Her email address is teresa .heiss@ist.ac.at.

Sarah Percival is a postdoc at Michigan State University. Her email address is sarah.b.perciva1@gmai1.com.

Lori Ziegelmeier is a professor of mathematics at Macalester College. Her email address is 1ziege11@maca 1ester. edu.

DOI: https://dx.doi.org/10.1090/noti 2349 


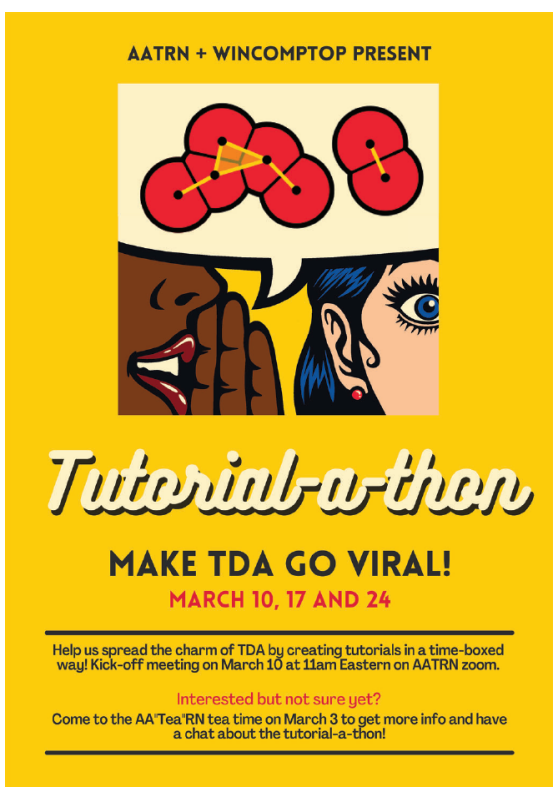

Figure 1. Poster to attract attention to the tutorial-a-thon, sent out as an advertisement to various mailing lists and forums. benefit of reducing emails and allowing participants to network. The videos we received were lightly moderated (by each small group of size three), and vastly exceeded our expectations in terms of their quality.

In this article, we describe tutorial-a-thons in more detail, with the hope that we will inspire you to consider running similar events in your research community, home department, or outreach group. See the webpage https:// sites.google.com/view/aatrn-tutorial-a-thon for our first tutorial-a-thon, which contains links to the contributed YouTube videos, and which also contains all of our organizational materials.

\section{What Does a Tutorial-a-thon Look Like for Participants?}

During the first week of the tutorial-a-thon, participants meet on Zoom to hear about the logistics of the process from the organizers. Then, using a sticky-note application called Padlet, each participant proposes a topic they would like to explain. Participants' proposals then get shuffled around, matching topics that are similar in some sense, until groups of size three are formed. Afterwards, participants split into breakout rooms to connect with their group and exchange contact information.

Each group has the freedom to choose a time, approximately one week later, to give trial-run tutorials and to receive constructive feedback from each other. Recording the trial run is necessary to test the technology, and to force participants to give an honest trial run! In most instances, groups meet one more time a week later on Zoom to incorporate feedback given by group members and to record the final videos. After this meeting, each participant submits their video to the organizers who then post it to YouTube.

In sum, the time commitment for each tutorial-a-thon participant is less than three hours (not counting the time to develop the materials to present a tutorial).

There are many benefits to this format, both for the participants and for the organizers:
- Soft deadlines and social reminders are in placeso videos get submitted in a timely fashion!

- Videos are moderated by each small group, and thus, the organizers do not need to peer review hours of videos themselves.

- Organizers provide tips and hints prior to the event. This streamlines the process, particularly with regard to technology. The organizers also run online office hours during a common meeting time in case there are questions.

- Meeting other participants creates social and networking opportunities, enhancing connections and a sense of belonging within the community.

- Early-career voices get shared.

- All of the videos get posted to a common community YouTube channel, which improves each video's visibility.

\section{Frequently Asked Questions from Participants}

What follows are frequently asked questions from participants, and responses that you might give as an organizer!

I'm confused-does my group make a single video, or does each participant make their own video? Each participant makes their own short video, which need not be thematically related to their group members' videos.

Is a 15-minute video okay? Yes. We tell folks their video should be at most 10 minutes, expecting to receive videos in the range of 5-15 minutes.

Should the videos be ordered? Should the topics be organized? This is not how we've done things so far. Each person has a story to tell-but their mathematical tidbit may not fit well in an ordered list. It is possible to provide a link to a related tutorial in the description of a YouTube video. We recommend an unordered set of videos for your first tutorial-a-thon, but we may experiment with more organized (or even ordered) video topics in follow-up events.

What if my topic is the same as somebody else's? That's okay! We have found that different viewpoints on the same topic are useful for folks learning a new area.

What if I want to record my video off-line (not during a group meeting)? That's fine! We still encourage you to meet with your small group, if possible, to give and receive feedback on your group's trial runs.

The final two questions are infrequently asked, but still worth considering beforehand.

What if I'm concerned that one of my group member's tutorial videos is not appropriate? Please let us know, and thank you for the heads up. If they submit a tutorial video that is not appropriate for the channel, then we will contact them (and perhaps work with them to address the problem).

I gave you permission to post the video, but I've now changed my mind and would like the video taken down. Is that possible? Yes, we're happy to remove your video at your request at any time. 


\section{Making Mosaics from Images}

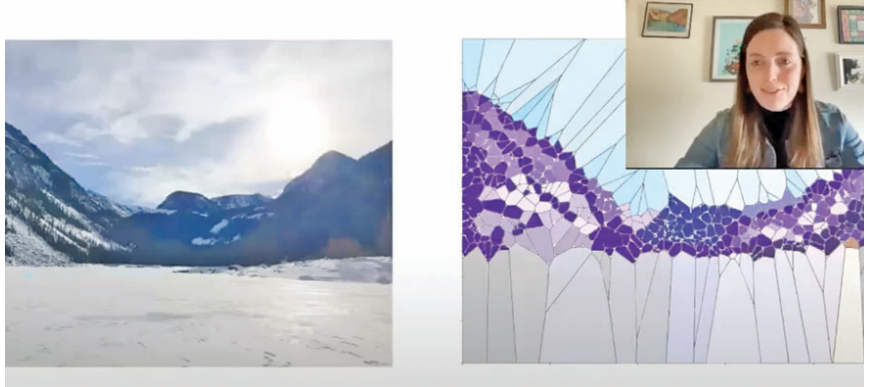

Figure 2. Screenshot from the tutorial How to Create Mosaics from Images by Robin Belton.

\section{How Do You Run a Tutorial-a-thon?}

Below we describe some tips that we have learned from running our first tutorial-a-thon, which was cohosted by the Applied Algebraic Topology Research Network (AATRN) and Women in Computational Topology (WinCompTop) communities.

Like with any other successful project, it is essential to start planning well ahead of time and to have a good team. Our team profited in large part from having graduate student, postdoctoral, and faculty organizers. This not only drew in diverse ideas, but also participants from a wide range of career levels.

Once your team has agreed on a rough structure for your tutorial-a-thon, we recommend that you prepare some advertising material-marketing is what draws people in! Think about how and where to advertise. We advertised with a poster and a website, via relevant mailing lists and forums; see Figure 1.

Our next tip is to do a trial run with your organizational team-i.e., have each member of your organizational team prepare a short tutorial. This not only gives you a first-hand impression of the outcome of the project and the technology tools you may recommend that participants use, but also provides you with sample videos that you can share to help participants better grasp the idea of the project.

Now it's time to kick off the project! After an introductory presentation and a Q\&A session, you need to sort the participants into small groups. You could do this off-line before your second meeting-for example, creating groups of size three at random, or based on self-reported experience levels, or based on the topics of their tutorials.

We instead chose the chaotic route of a live organic sorting procedure, using Zoom and an online sticky-note application called Padlet. Having had a rough estimate of how many participants to expect, we asked each participant to write their tutorial topic on a digital sticky note and move their sticky note around until everybody was matched in a group of three. At the same time, we brainstormed with those who were not yet sure about their topics, and helped out with the matching process. After the groups were formed, we sent these groups off into breakout rooms where the members of each group exchanged contact information and discussed the internal organization.

This chaotic matching process was a lot of fun, and a great success! However, if you decide to do this approach, we recommend you involve at least two people in the management of the matching process-one person moderates, one person does the actual matching and direction of participants, and keeps an eye on the chat.

With the participants matched most of your work is done! We kept in touch with our participants by emailing out weekly updates and holding office hours. To collect the tutorials, together with their titles, short descriptions, and the names of the authors, we used Google forms and the website We-Transfer. We uploaded all of the tutorials to the AATRN YouTube channel https://www. youtube . com/c/AppliedA7gebraicTopologyNetwork, to a tutorial-a-thon playlist.

One last tip-make sure you delete all personal information you collected about the participants after finishing the project.

If you are interested in running a tutorial-a-thon of your own (and we hope you are), please feel free to email the authors with any questions, or to arrange a time to chat.

\section{How Did Our First Tutorial-a-thon Go?}

Our first tutorial-a-thon in March 2021 was such a success that we decided to organize our second tutorial-a-thon six months after the first-and possibly many more in the future.

During our first tutorial-a-thon, we received 38 tutorials, from a very diverse pool of participants! Approximately half of them were done by PhD students, and the rest from postdocs, professors, and people outside of academia. We had participants from North America, South America, Europe, Africa, and Asia. The impressively high quality of all 38 videos shows that everyone-even a young $\mathrm{PhD}$ student-has topics they can explain exceptionally well. See Figures 2, 3, and 4 for example screenshots of three of these excellent tutorials.

In the first two months after posting, the videos already have received a lot of attention, and the number of views

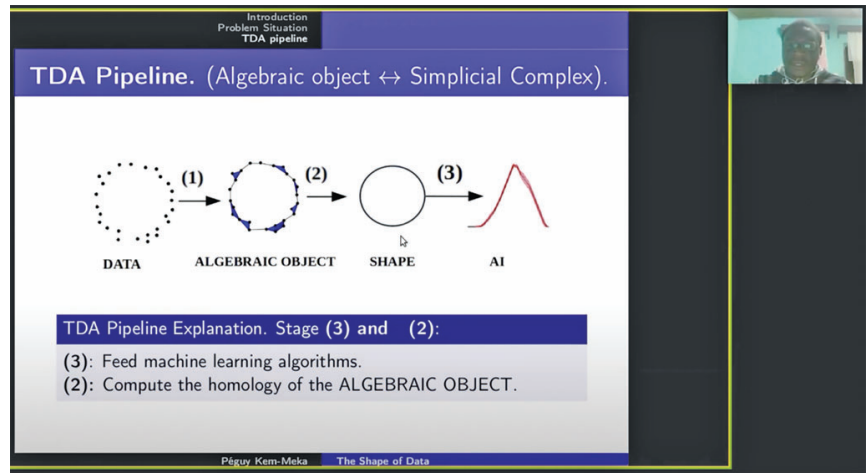

Figure 3. Screenshot from the tutorial The Shape of Data by Péguy Kem-Meka. 
is rising every day. By the time of the writing of this article, each tutorial has been viewed 372 times on average, and the median number of views per tutorial is 266 . Given the size of the applied topology community, this is truly remarkable. One of the tutorials has been viewed over 2400 times already.

The tutorials received very positive feedback from the community, both via email and via public (moderated) YouTube comments on the videos. Some of the YouTube viewers shared in their comments that with the help of this video, they finally understood something they had struggled with for a while. One enthusiastic viewer even asked if they could translate a video into Korean. The participants shared with us that making the videos was fun and a nice way to meet other researchers in applied topology. They also enjoyed watching the number of views of their own videos rise.

\section{Conclusion}

Before the advent of the internet, excellent oral explanations of mathematical topics could only reach the handful of people who were able to be in the same room as the presenter. With the prevalence of YouTube, not only are we able to reach anyone around the globe, but we are able to reach future mathematicians as well. Furthermore, by soliciting contributions from early-career researchers who may not yet have had the opportunity to teach, we are able to tap into a wealth of knowledge that may not otherwise be shared.

We hope online activities such as the tutorial-a-thon can help make mathematics more geographically and linguistically diverse. Because each group sets their own meeting time, participation in the tutorial-a-thon is not limited to researchers from any particular time zone. Furthermore, unlike traditional conferences, participation in the tutorial-a-thon does not require travel funding, which can be prohibitive, especially for researchers from geographically

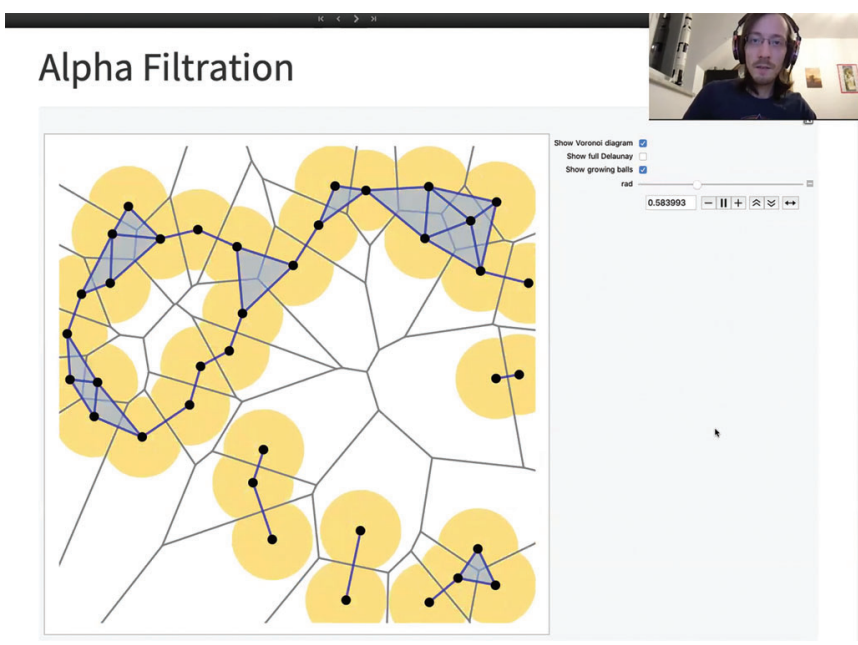

Figure 4. Screenshot from the tutorial Voronoi diagram, Delaunay and Alpha complexes: A Visual Intro by Ondřej Draganov. underresourced areas, as well as for undergraduate and graduate students. Additionally, because most conferences are held in English, future tutorial-a-thons could reach new audiences by soliciting videos in other languages.

The tutorial-a-thon format provides a platform for mathematicians from across the globe to meet and discuss mathematics in a low-pressure environment. Because participants at different career stages are encouraged to group together, we hope that our tutorial-a-thon provides a sense of belonging in the greater mathematical community, particularly for early-career researchers. Stay tuned for our second applied topology tutorial-a-thon in October and November 2021!

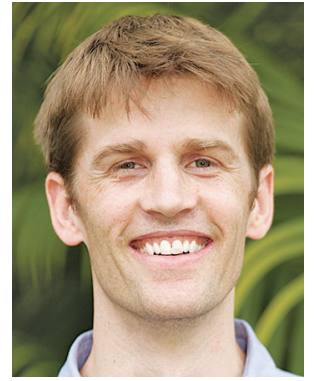

Henry Adams

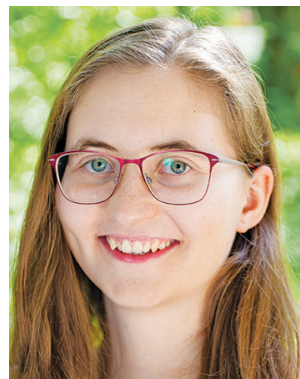

Teresa Heiss

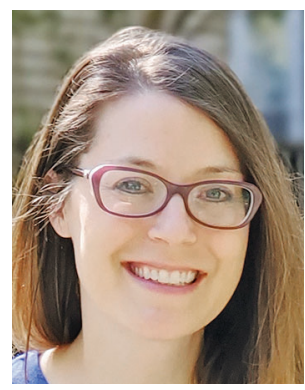

Lori Ziegelmeier

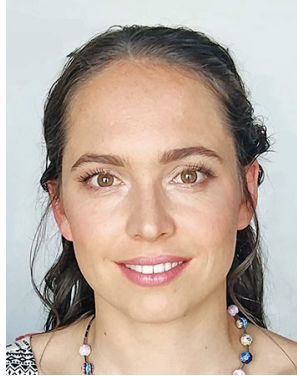

Hana Dal Poz Kouřimská

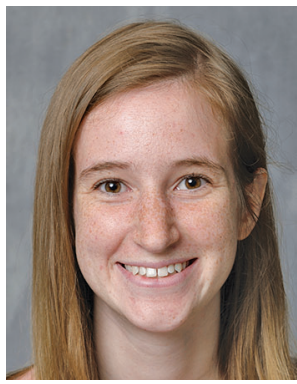

Sarah Percival

\section{Credits}

Figure 1 is courtesy of Hana Dal Poz Kouřimská.

Figures 2-4 are courtesy of the authors.

Photo of Henry Adams is courtesy of Ewo Harrell Adams.

Photo of Hana Dal Poz Kouřimská is courtesy of Simone Dal Poz.

Photo of Teresa Heiss is courtesy of Michael Heiss.

Photo of Sarah Percival is courtesy of Rebecca Lank.

Photo of Lori Ziegelmeier is courtesy of Michael Neuberg. 